\title{
Carbonetos de WC com fase ligante Co-Ni, comportamento da dureza e resistência à ruptura transversal em função da adição de SiC- $\alpha$ e seus efeitos na temperatura de sinterização
}

\author{
Daniel Rodrigues ${ }^{1}$ \\ Francisco Yastami Nakamoto ${ }^{2}$ \\ Givanildo Alves dos Santos ${ }^{2}$ \\ Fabio Miranda ${ }^{3 *}$ (1) \\ Gilmar Ferreira Batalha ${ }^{3}$ \\ Laerte Fernandes ${ }^{3}$
}

\section{Resumo}

A vantagem de substituir o WC $\left(15,67 \mathrm{~g} / \mathrm{cm}^{3}\right)$ pelo $\mathrm{SiC}-\alpha\left(3,21 \mathrm{~g} / \mathrm{cm}^{3}\right)$ é o custo da matéria-prima e a baixa densidade do carboneto. A substituição do $\mathrm{Co}\left(8,90 \mathrm{~g} / \mathrm{cm}^{3}\right)$ pelo $\mathrm{Ni}\left(8,91 \mathrm{~g} / \mathrm{cm}^{3}\right)$, é devido à escassez da matéria-prima, restrições ao uso devido a exposição ocupacional e o preço comercial. Em relação aos experimentos realizados neste estudo, foram preparadas 3 misturas, sendo a primeira, a partir de $\mathrm{WC}-11(\mathrm{Co}, \mathrm{Ni})$ e as demais reforçadas com adição de teores $0,6 \% \mathrm{e}$ $1,2 \%$ de SiC- $\alpha$, em peso, que foram produzidas pela técnica de metalurgia do pó convencional. Os compactados a verde foram sinterizados em fornos Sinter HIP, sinterização a vácuo em 1.380 e $1.420^{\circ} \mathrm{C}$ durante 60 minutos de estabilização e submetidos a uma pressão de 20 bar com nitrogênio. Os resultados apresentaram que com o excesso de carbono livre presente matéria-prima e mais o aumento da temperatura de sinterização influenciaram fortemente no crescimento do grão de WC e no surgimento de porosidades que consequentemente afetaram as propriedades mecânicas das ligas de metal duro.

Palavras-chave: Metalurgia do pó; Metal duro; SiC- $\alpha$; Sinterização; Microestrutura e propriedades.

\section{WC cemented carbides with based Co-Ni binder phase - hardness and transverse rupture strength behaviour as a function of $\mathrm{SiC}-\alpha$ additions and their effects on the sintering temperature}

\begin{abstract}
The advantage of replacing the WC $\left(15.67 \mathrm{~g} / \mathrm{cm}^{3}\right)$ with $\mathrm{SiC}-\alpha\left(3.21 \mathrm{~g} / \mathrm{cm}^{3}\right)$ is the cost of the raw material and the low density. The substitution of $\mathrm{Co}\left(8.90 \mathrm{~g} / \mathrm{cm}^{3}\right)$ for $\mathrm{Ni}\left(8.91 \mathrm{~g} / \mathrm{cm}^{3}\right)$, it is due to shortages of raw material, restrictions on use due to occupational exposure and the commercial price. In relation to the experiments carried out in this work, 3 mixtures were prepared, the first being from WC-11 $(\mathrm{Co}, \mathrm{Ni})$ and the others reinforced with the addition of $0.6 \%$ and $1.2 \%$ of $\mathrm{SiC}-\alpha$, by weight, which were produced by the conventional powder metallurgy technique. The green compacted ones were sintered in SinterHIP ovens, vacuum sintered at 1,380 and $1,420^{\circ} \mathrm{C}$ for 60 minutes of stabilization and subjected to a pressure of 20 bar with nitrogen. The results showed that, with the excess of free carbon present in the raw material and the increase in the sintering temperature, strongly influence the growth of the WC grain, in the appearance of porosities that consequently affected the mechanical properties of hardmetal.
\end{abstract}

Keywords: Powder metallurgy; Hardmetals; SiC- $\alpha$; Sintering; Microstructure and properties.

${ }^{\prime}$ BRATS Filtros Sinterizados e Pós Metálicos, São Paulo, Cajamar, SP, Brasil. ${ }^{2}$ Departamento de Engenharia, Instituto Federal de São Paulo, IFSP, São Paulo, SP, Brasil.

${ }^{3}$ Departamento de Engenharia Mecatrônica e de Sistemas Mecânicos, Escola Politécnica, Universidade de São Paulo, USP, São Paulo, SP, Brasil.

*Autor correspondente: fabio.miranda@usp.br 


\section{Introdução}

O metal duro tem sido usado como ferramentas de corte, na usinagem, desde a década de 1920. Conhecido como um material cerâmico, é processado pela rota convencional da metalurgia do pó, sendo fabricado a partir de pós-metálicos, carbonetos de tungstênio (WC) e cobalto (Co), podendo ser utilizado novos ligantes alternativos, especialmente o níquel (Ni) ou ferro (Fe), a combinação de dureza e ductilidade fornece propriedades mecânicas superiores a outras ligas [1]. Em 1933, foi desenvolvido uma liga WC-Ni com a marca RENIK, como substituto da liga WC-Co, pela empresa Moscow Plant of Rare Chemical Elements, para munições e armamentos durante a $2^{\mathrm{a}}$ Guerra Mundial [2]. No entanto, não se sabe ainda se é possível usar essas novas ligas de metal duro definitivamente para o torneamento de aços e como eles se comportarão durante a aplicação. Sabe-se que a fase ligante desempenha um papel importante no comportamento de deformação do metal duro, e é importante investigar essas novas ligas com fase ligante alternativos, $\mathrm{Ni}$ e Fe, ou combinados, e comparar com as ligas WC-Co [3]. Existem três motivos principais para a substituição do Co pelo Ni em metais duro: (1) a matéria-prima Co tem preço comercial mais elevado, (2) quando liberado no ambiente de trabalho, na forma de aerodispersóides, Co resulta em doenças ocupacionais e (3) o Ni possui maior resistência à corrosão do que o Co [4]. O presente trabalho visa analisar o comportamento do WC-11(Co, Ni) na proporção 1,5:1 de Co:Ni, respectivamente, durante a sinterização, com a adição de carboneto de silício (SiC- $\alpha$ ), que é um material cerâmico amplamente utilizado na indústria de refratários, fundição e

Tabela 1. Composição química de cada mistura, \% em massa

\begin{tabular}{ccccc}
\hline Descrição & WC & Ni & Co & SiC- $\boldsymbol{\alpha}$ \\
\hline Liga (1) & 89,0 & 7,0 & 4,0 & 0,0 \\
Liga (2) & 88,5 & 7,0 & 4,0 & 0,6 \\
Liga (3) & 87,8 & 7,0 & 4,0 & 1,2 \\
\hline
\end{tabular}

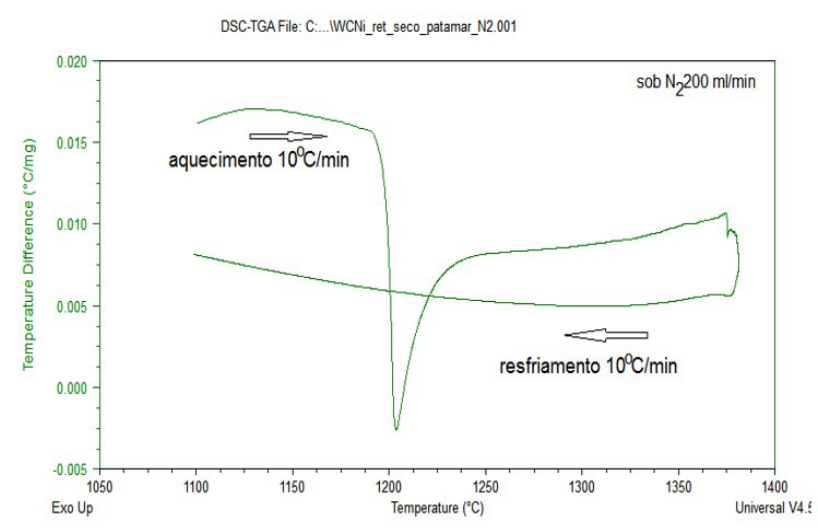

(a) de abrasivos e a sua formação ocorre em duas fases, através da redução carbotérmica da sílica: I) a $1700^{\circ} \mathrm{C}$ é formado o $\mathrm{SiC}-\beta$ (com estrutura cristalina cúbica) e gerado monóxido de carbono (CO); e II) a aproximadamente $2.100{ }^{\circ} \mathrm{C}$ a estrutura do $\mathrm{SiC}-\beta$ é transformado em $\mathrm{SiC}-\alpha$, com predominância de estrutura cristalina hexagonal [5].

\section{Materiais e métodos}

Neste trabalho foram utilizados: I) WC com tamanho médio $=3,5 \mu \mathrm{m}$ (Fisher); II) pó de carboneto de Silício, com a composição química $96,8 \%$ SiC- $\alpha, 0,5 \% \mathrm{C}, 1,8 \%$ Si, $0,48 \% \mathrm{Fe}, 0,25 \% \mathrm{Al}$, formato angular, distribuição granulométrica: $\mathrm{D}_{3}=7,0 \mu \mathrm{m}$ máximo; $\mathrm{D}_{50}=2,5$ a $3,0 \mu \mathrm{m}$ e $\mathrm{D}_{94}=1,0$ mínimo $\mu \mathrm{m}(80 \%)$, \#1200 mesh, com estrutura cristalina SiC- $\alpha\left(3,21 \mathrm{~g} / \mathrm{cm}^{3}\right)$, para aplicação de abrasivos; e III) Co e Ni (carbonila), 400 mesh. Foram preparadas três misturas, apresentada na Tabela 1, por balanço de massa, via moinho de Atrittor de alta energia, com álcool isopropílico, por 3 horas.

Foram realizadas para análise térmica diferencial (DSC) e dilatometria, das ligas (1) WC-11(Co, Ni) e (3) $\mathrm{WC}-11(\mathrm{Co}, \mathrm{Ni})-1,2 \mathrm{SiC}-\alpha$, para saber as mudanças de fase e a determinação de valores calóricos como calor de fusão, dados obtidos nas Figuras 1a e 1b, sob 200 mililitros por minutos de nitrogênio, à uma taxa de aquecimento de $10{ }^{\circ} \mathrm{C}$ por minutos, há uma formação da fase líquida $(\mathrm{Co}, \mathrm{Ni})$ a partir de $1.200^{\circ} \mathrm{C}$, processo endotérmico. Na temperatura de $1.380{ }^{\circ} \mathrm{C}$, aproximadamente, ocorre a fase liquida por completo da liga WC-11(Co, Ni).

Após o ensaio de DSC, Figurala, foi realizado o ensaio de dilatometria linear, Figura $1 \mathrm{~b}$ para as misturas $\mathrm{WC}-11(\mathrm{Co}, \mathrm{Ni})$ e WC-11(Co, Ni) $-1,2 \mathrm{SiC}-\alpha$.

Essas mudanças, fornecem informações a respeito dos mecanismos de sinterização desses compósitos (sinterização por fase sólida e líquida), isto deve ser acompanhado pela

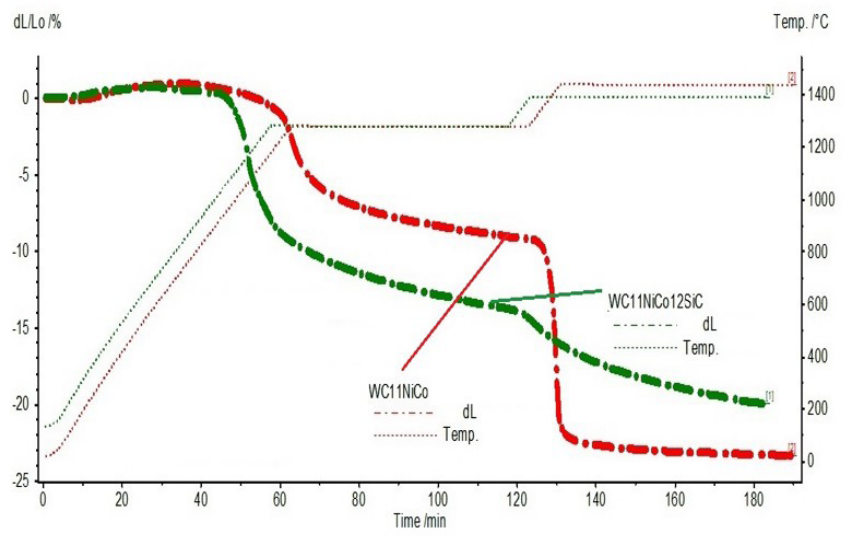

(b)

Figura 1. (a): DSC para as misturas de WC-11(Co, Ni). (b): Dilatometria das misturas WC-11(Co, Ni) à $1420^{\circ} \mathrm{C}$ e WC-11(Co, Ni) $1,2 \mathrm{SiC}-\alpha-\alpha$ à $1380^{\circ} \mathrm{C}$. 
observação das microestruturas no microscópio óptico (MO), apresentadas mais adiante nas Figuras 3abc para $1380^{\circ} \mathrm{C}$ e Fig. 3def para $1420^{\circ} \mathrm{C}$. Após os ensaios de DSC e Dilatometria, foram compactados dois corpos de prova para cada mistura (1), (2) e (3), com $200 \mathrm{MPa}$, por prensagem uniaxial de ação simples numa matriz de aço dimensionada para atingir as medidas sinterizadas. Os compactados a verde foram sinterizados em um forno SinterHIP, a vácuo. A Figura 2a apresenta o ciclo térmico para as temperaturas de 1.380 e $1.420{ }^{\circ} \mathrm{C}$, com estabilidade de 60 minutos.
Após a sinterização, os corpos de prova foram retificados nos quatros lados com rebolo resinóide diamantado para atingir um grau de rugosidade fino entre $\mathrm{N} 4(\mathrm{Ra} 0,2 \mu \mathrm{m}) \mathrm{e}$ N6 $(\operatorname{Ra} 0,8 \mu \mathrm{m})$, para a realização de resistência à ruptura transversal (TRS), em três pontos, Figura 2b. As medidas das densidades dos sinterizados, obtidas pelo princípio de ensaio de Arquimedes, com base na norma MPIF 95. Os ensaios de dureza aparente, Rockwell na escala A, foram conduzidos em um durômetro modelo Galileo, aplicando uma carga de $60 \mathrm{kgf}$, com ponta cônica de diamante com $120^{\circ}$,

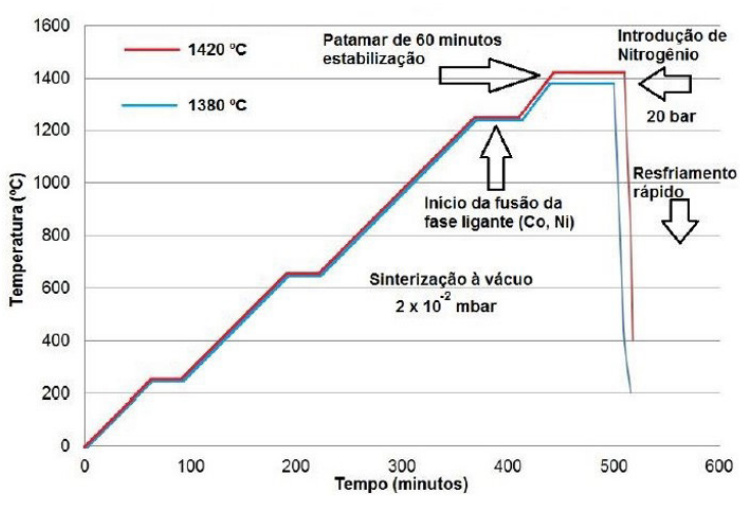

(a)

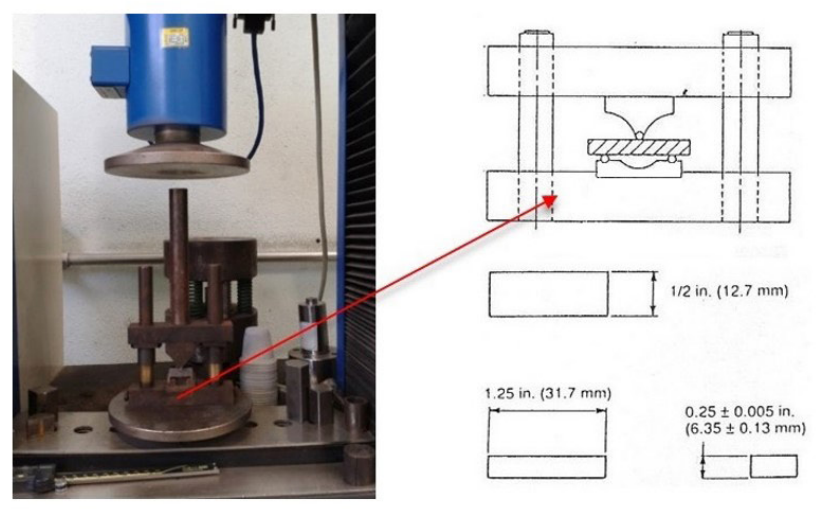

(b)

Figura 2. (a): Ciclo de Sinterização dos corpos de prova para o ciclo $1\left(1.380^{\circ} \mathrm{C}\right)$ e ciclo $2\left(1.420^{\circ} \mathrm{C}\right)$. (b): Equipamento para o ensaio de TRS. Marca: EMIC DL 10000 e desenho esquemático do dispositivo de TRS por 3 pontos e corpo de prova.

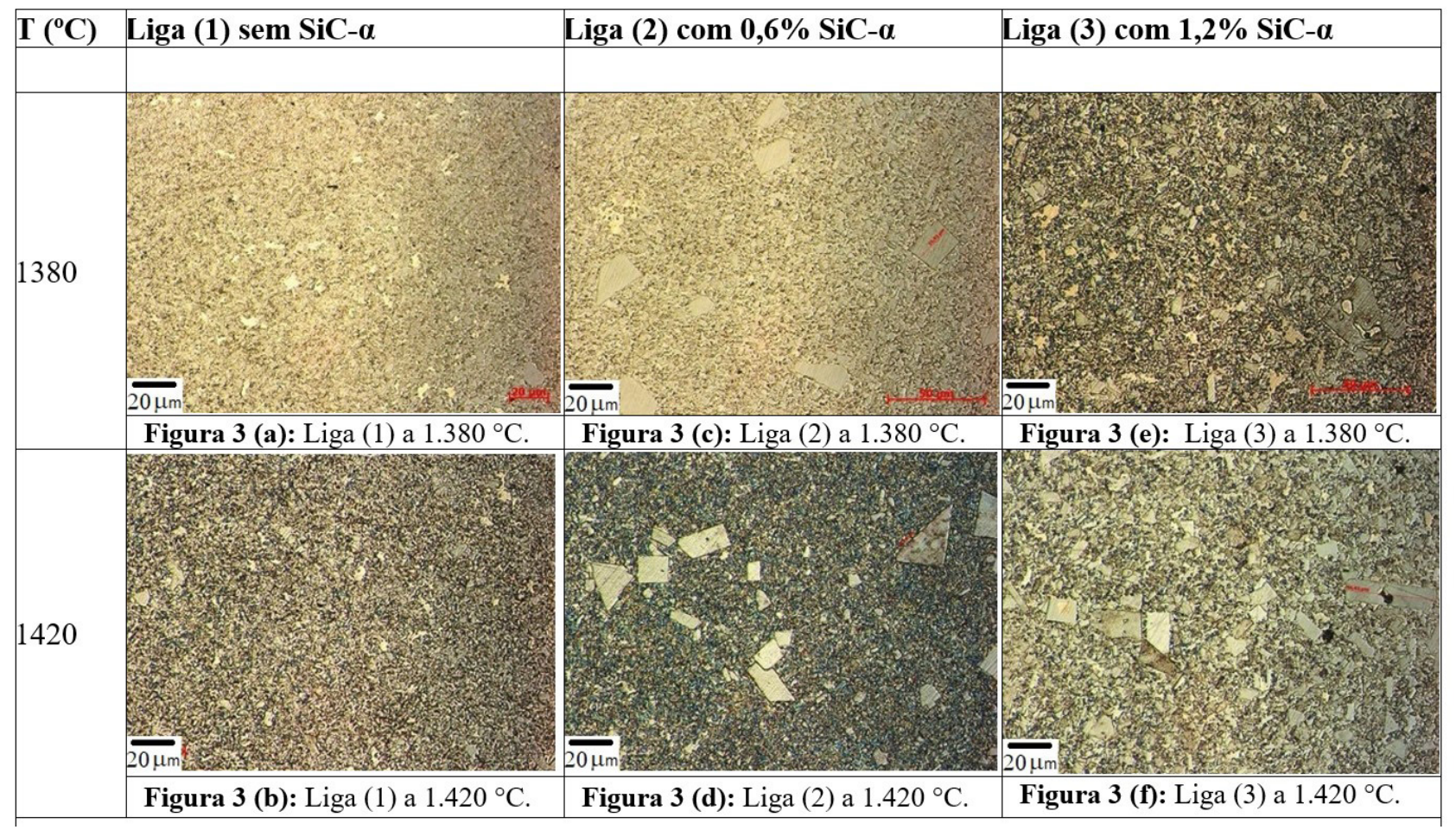

Figuras 3. (a) a (f): Microestruturas das amostras em função da adição de \% SiC- $\alpha$ e em função da temperatura de sinterização - Ataque eletrolítico (3V) com reagente de Murakami (3V) - Microscópio óptico - ampliada 500x. 
conforme as normas ASTM B294 [6] e ISO 3738 [7]. Neste procedimento foram realizadas 10 análises em cada corpo de prova para fins de confiabilidade dos resultados obtidos.

A caracterização microestrutural dos corpos de prova sinterizados foi feita por meio de microscópio óptico (MO) e microscopia eletrônica de varredura (MEV/ EDS). $\mathrm{Na}$ quantificação do tamanho de grão médio do WC, foi utilizado o programa Axio Vision Release 4.9.1 para análise de intercepto linear médio juntamente com intercepto por uso de linhas circulares, um método de intercepção automático, de acordo com a norma ASTM E112 [8], que é recomendado para procedimento de interceptos para todas as estruturas que partem de forma equiaxial uniforme. No ensaio de TRS, as amostras foram embasadas nas normas ASTM B-406-76 [9], utilizando uma máquina de ensaio universal EMIC, devidamente calibrada, apresentada na Figura $2 b$, a uma taxa de carga de $0,5 \mathrm{~mm} /$ minuto à temperatura ambiente.

\section{Resultados}

As Figuras 3a a $3 \mathrm{f}$, apresentam as micrografias das ligas (1), (2) e (3); as partículas de reforço de $\mathrm{SiC}-\alpha$ foram distribuídas homogeneamente na fase ligante $(\mathrm{Co}$, $\mathrm{Ni})$, região clara em torno das partículas angulares. As partículas angulares ou triangulares, cinza escuras, na micrografia representam as partículas de WC e a fase de reforço $\mathrm{SiC}-\alpha$, notando-se difusão, marrom claro, por total na liga (3) à $1.380^{\circ} \mathrm{C}$. Nessas micrografias, ampliadas 500 vezes, percebe-se a alteração dos grãos de WC, em função da temperatura de sinterização de 1.380 para $1.420^{\circ} \mathrm{C}$ e da adição de $\mathrm{SiC}-\alpha$, o crescimento anormal, significativo para baixos teores de $\mathrm{SiC}-\alpha$.

A variação das cores nas micrografias demonstra que os tempos de ataque dos corpos de prova foram diferentes, variando de 3 a 5 segundos, o que ocasionou no seu escurecimento, quando comparados, por exemplo, as Figuras 3c e 3d. Na Figura 4a, é apresentada a EDS qualitativa por espectroscopia de dispersão de energia e apresentou a predominância dos grãos de WC (cinza claro), Ni e Co (região cinza escuro), de forma geral, uma microestrutura homogênea com grãos bem distribuídos para a liga (1) WC-11(Co, Ni) sem adição de SiC- $\alpha$. A Figura $4 \mathrm{~b}$ apresenta a microestrutura da liga (2) a $1420^{\circ} \mathrm{C}$, microscópio ótico ampliado 1000 vezes, percebe-se a precipitação de carbono livre em excesso entorno dos particulados de WC.

A análise por EDS da liga (3) foi realizada, conforme apresentada na Figura 5 a e 5 b, nesta região, foram evidenciadas as fases $\mathrm{W}-\mathrm{Ni}, \mathrm{W}-\mathrm{Si}, \mathrm{Co}$ e $\mathrm{Fe}$. A presença de tungstênio e silício no espectro de EDS da matriz, Figura 5b, indica que ocorreu dissolução das partículas na fase ligante $(\mathrm{Co}, \mathrm{Ni})$.

A Tabela 2 apresenta a composição química da região pertinente às Figuras $5 \mathrm{a}$ e $5 \mathrm{~b}$ por meio de análise química semiquantitativa EDS em microscópio eletrônico de varredura.

A Tabela 3 apresenta os resultados obtidos, a dureza, densidade, contração volumétrica, análise do tamanho do grupo e a tensão de ruptura transversal, das ligas.

Os valores das durezas e das densidades dos corpos de provas, são apresentadas nas Figuras 6a e b. O gráfico de TRS é apresentado na Figura $7 \mathrm{~b}$, os valores de resistência diminuíram com o aumento do teor de SiC- $\alpha$, para as temperaturas de 1.380 e $1.420^{\circ} \mathrm{C}$.

Comparando os resultados obtidos da Figura 7b, com os resultados de Correa et al. [4], os valores ficaram muito abaixo do esperado. Para um compósito WC-10Ni a TRS é próximo de $1.400 \mathrm{MPa}$, para um compósito com WC-10Co, TRS=1.700 MPa e WC-10 (Ni-4,1Si) TRS = 2.000 MPa.

A presença de porosidade aparente foi analisada no microscópio ótico, ampliada 100 vezes, apresentada nas Figuras 8a a 8f, é um fator importante a ser analisado que pode influenciar nas propriedades do metal duro,

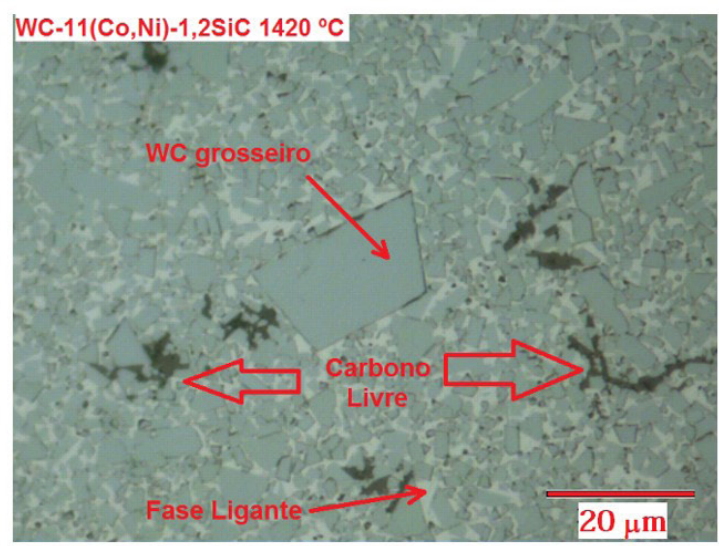

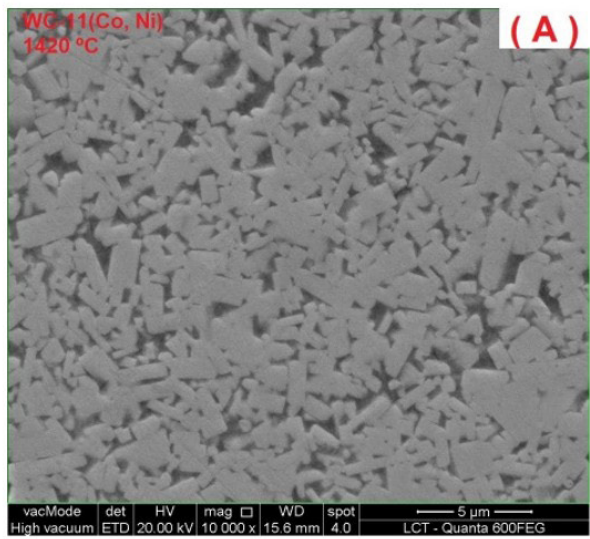

(a) (b)

Figura 4. (a): EDS da microestrutura da liga (1). (b): Liga (2) a $1.420^{\circ} \mathrm{C}$, revelação do Carbono livre. 
principalmente na tensão de ruptura transversal. As ligas

(2) e (3) apresentaram porosidades do Tipo B (poros acima de $20 \mu \mathrm{m})$ e C (carbono livre).

\section{Discussão}

Os diagramas de fase são úteis na identificação de parâmetros de solubilidade e outras características importantes

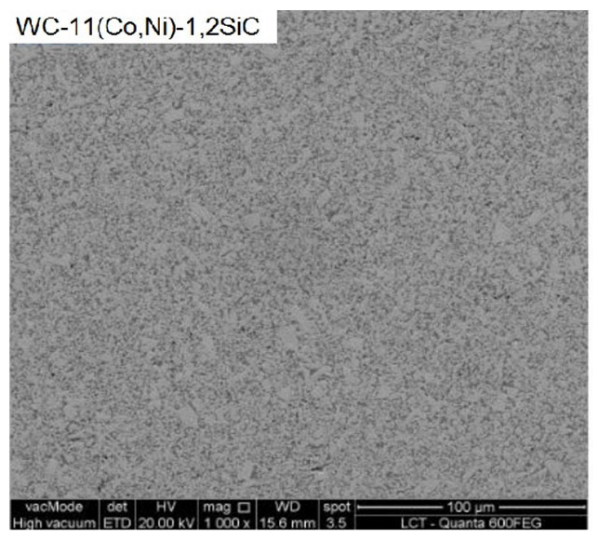

(a)

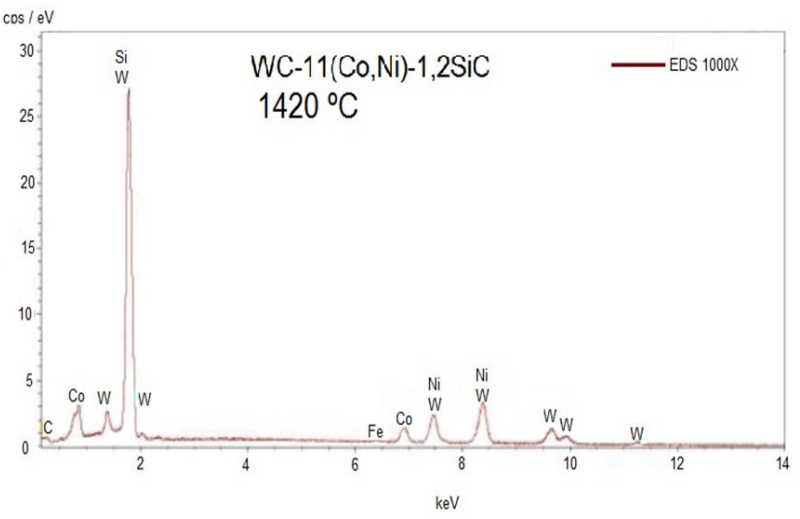

(b)

Figuras 5. (a): MEV da liga (2) a $1420^{\circ} \mathrm{Ce}$ (b) análise química semiquantitativa por espectrometria de dispersão de energia (EDS) da mesma liga.

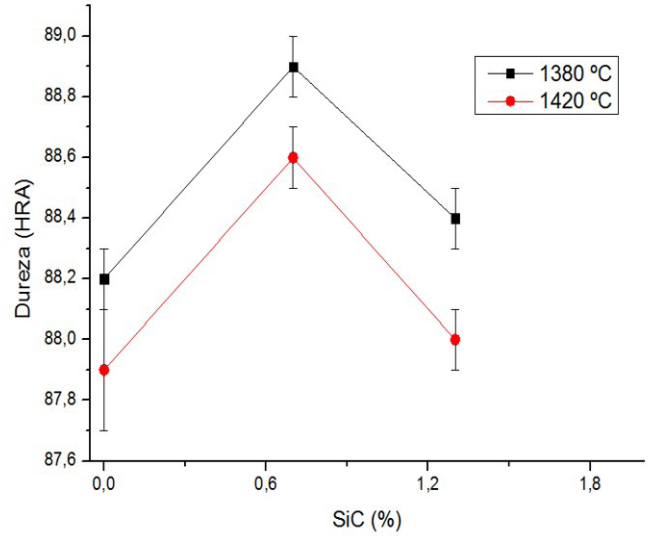

(a)

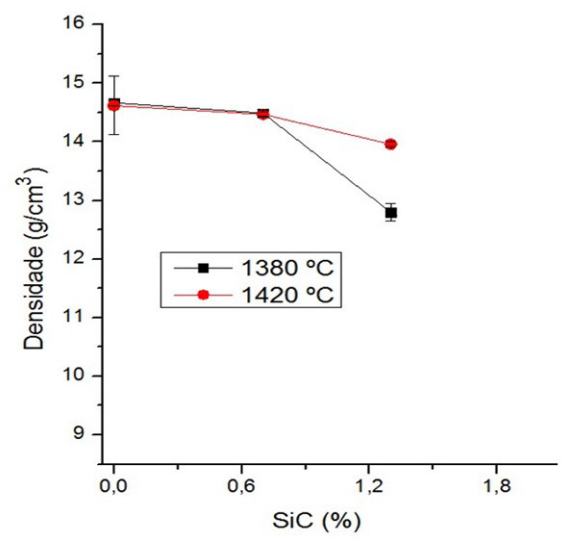

(b)

Figura 6. (a): Dureza dos compósitos de WC-11(Co, Ni) em função da adição de SiC- $\alpha$ e da temperatura. (b): Massa específica das ligas em função da adição de $\mathrm{SiC}-\alpha$ e da temperatura.

Tabela 2. Composição química da região EDS, Figura 5a, WC-11(Co, Ni) $-1,2 \mathrm{SiC}-\alpha \mathrm{T}=1420^{\circ} \mathrm{C}$

\begin{tabular}{cccccccc}
\hline Amostra/Região & $\mathbf{C}$ & $\mathbf{S i}$ & $\mathbf{C r}$ & $\mathbf{F e}$ & $\mathbf{C o}$ & $\mathbf{N i}$ & $\mathbf{W}$ \\
\hline EDS 1000x & $5,23 \%$ & $2,97 \%$ & - & $0,55 \%$ & $5,87 \%$ & $12,02 \%$ & $73,36 \%$ \\
\hline
\end{tabular}

Tabela 3. Resultados obtidos das misturas WC- $11(\mathrm{Co}, \mathrm{Ni})-\mathrm{T}=1380^{\circ} \mathrm{C}$ e $1420^{\circ} \mathrm{C}$

\begin{tabular}{|c|c|c|c|c|c|c|}
\hline Liga & $\mathrm{T}\left({ }^{\circ} \mathrm{C}\right)$ & $\begin{array}{c}\text { Dureza Aparente } \\
\text { (HRA) }\end{array}$ & $\begin{array}{c}\text { Densidade } \\
\left(\mathrm{g} / \mathrm{cm}^{3}\right)\end{array}$ & $\begin{array}{c}\text { ContraçãoVol. } \\
(\%)\end{array}$ & $\begin{array}{l}\text { Tamanho de grão } \\
\text { médio WC }(\mu \mathrm{m})\end{array}$ & $\operatorname{TRS}\left(\mathbf{N} / \mathbf{m m}^{2}\right)$ \\
\hline \multirow[t]{2}{*}{ (1) $0,0 \% \mathrm{SiC}$} & $1380^{\circ} \mathrm{C}$ & $88,2 \pm 0,3$ & $14,65 \pm 0,02$ & $46,9 \pm 1,15$ & $4,4 \pm 0,6$ & $1247 \pm 114$ \\
\hline & $1420^{\circ} \mathrm{C}$ & $87,9 \pm 0,3$ & $14,69 \pm 0,02$ & $45,8 \pm 1,03$ & $5,0 \pm 0,5$ & $1190 \pm 70$ \\
\hline \multirow[t]{2}{*}{ (2) $0,6 \% \mathrm{SiC}$} & $1380^{\circ} \mathrm{C}$ & $88,9 \pm 0,3$ & $14,43 \pm 0,03$ & $37,7 \pm 0,4$ & $4,8 \pm 0,6$ & $695 \pm 10$ \\
\hline & $1420^{\circ} \mathrm{C}$ & $88,6 \pm 0,3$ & $14,54 \pm 0,03$ & $38,7 \pm 1,44$ & $5,2 \pm 0,7$ & $360 \pm 10$ \\
\hline \multirow[t]{2}{*}{ (3) $1,2 \% \mathrm{SiC}$} & $1380^{\circ} \mathrm{C}$ & $88,4 \pm 0,3$ & $13,82 \pm 0,6$ & $28,7 \pm 0,44$ & $5,7 \pm 0,3$ & $420 \pm 50$ \\
\hline & $1420^{\circ} \mathrm{C}$ & $88,0 \pm 0,3$ & $14,10 \pm 0,6$ & $29,7 \pm 1,87$ & $6,2 \pm 0,3$ & $412 \pm 92$ \\
\hline
\end{tabular}


como os pontos de fusão dos elementos químicos. Um diagrama de fase WC por fase líquida ( $\mathrm{Fe}, \mathrm{Co}, \mathrm{Ni})$ é apresentado na Figura 9. Há um ponto de eutético no diagrama $\mathrm{WC}-(\mathrm{Fe}, \mathrm{Co}$, $\mathrm{Ni}$ ), sendo favorável para a fusão da fase ligante (Fe, Co, Ni), devido à grande redução da temperatura de sinterização com a formação da fase líquida, aproximadamente $1.300^{\circ} \mathrm{C}$ [10].

A formação de compostos intermetálicos, ou precipitados, na fase líquida, no geral é desfavorável para a diminuição da temperatura no ponto eutético. Na região do diagrama de fase alfa + Líquido, Figura 9, na sinterização por fase liquida, ocorrem a dissolução parcial dos particulados de WC e SiC- $\alpha$, o crescimento dos grãos de WC dará-se justamente por dissolução, difusão e precipitação na solução líquida, o resultado disto será a presença de grãos secundários de WC grosseiros e anormais em função do aumento da temperatura [11].

A Figura 10a, apresenta o Co alotrópico, e possui estrutura cristalina hexagonal compacta $(\mathrm{HC})$ até $427{ }^{\circ} \mathrm{C}$ $(\varepsilon-\mathrm{Co})$ e o Ni- $\alpha$ possui estrutura cúbica de face centrada (CFC). Para concentrações abaixo de $70 \%$ em massa de Co no Ni tem-se uma solução sólida $\mathrm{CFC}(\mathrm{Ni}, \alpha-\mathrm{Co})$, após transformação alotrópica $\mathrm{CFC}$, com a presença de $\mathrm{Ni}$ e

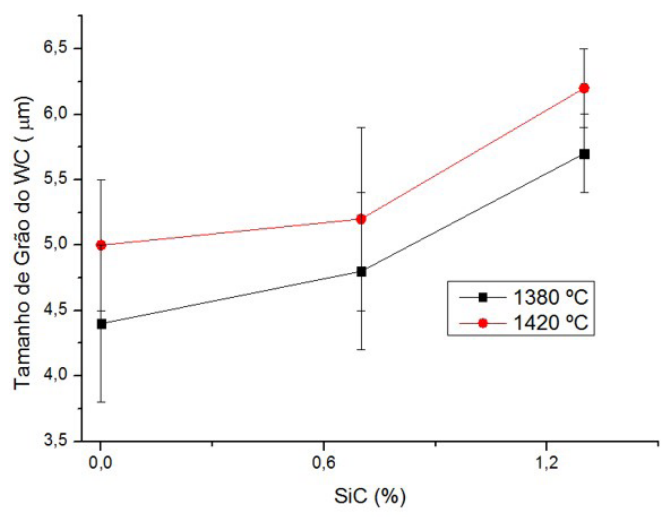

(a)

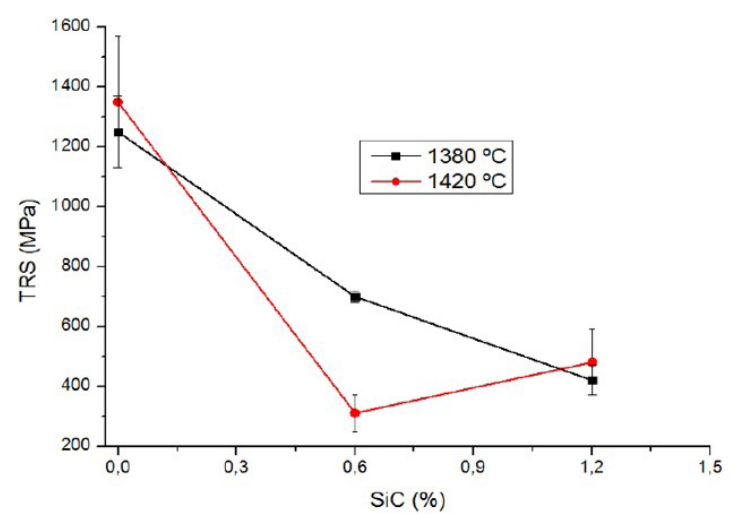

(b)

Figura 7. (a): Crescimento do grão WC em função da adição de SiC- $\alpha$ e da temperatura. (b): A influência do conteúdo de SiC- $\alpha$ no TRS.

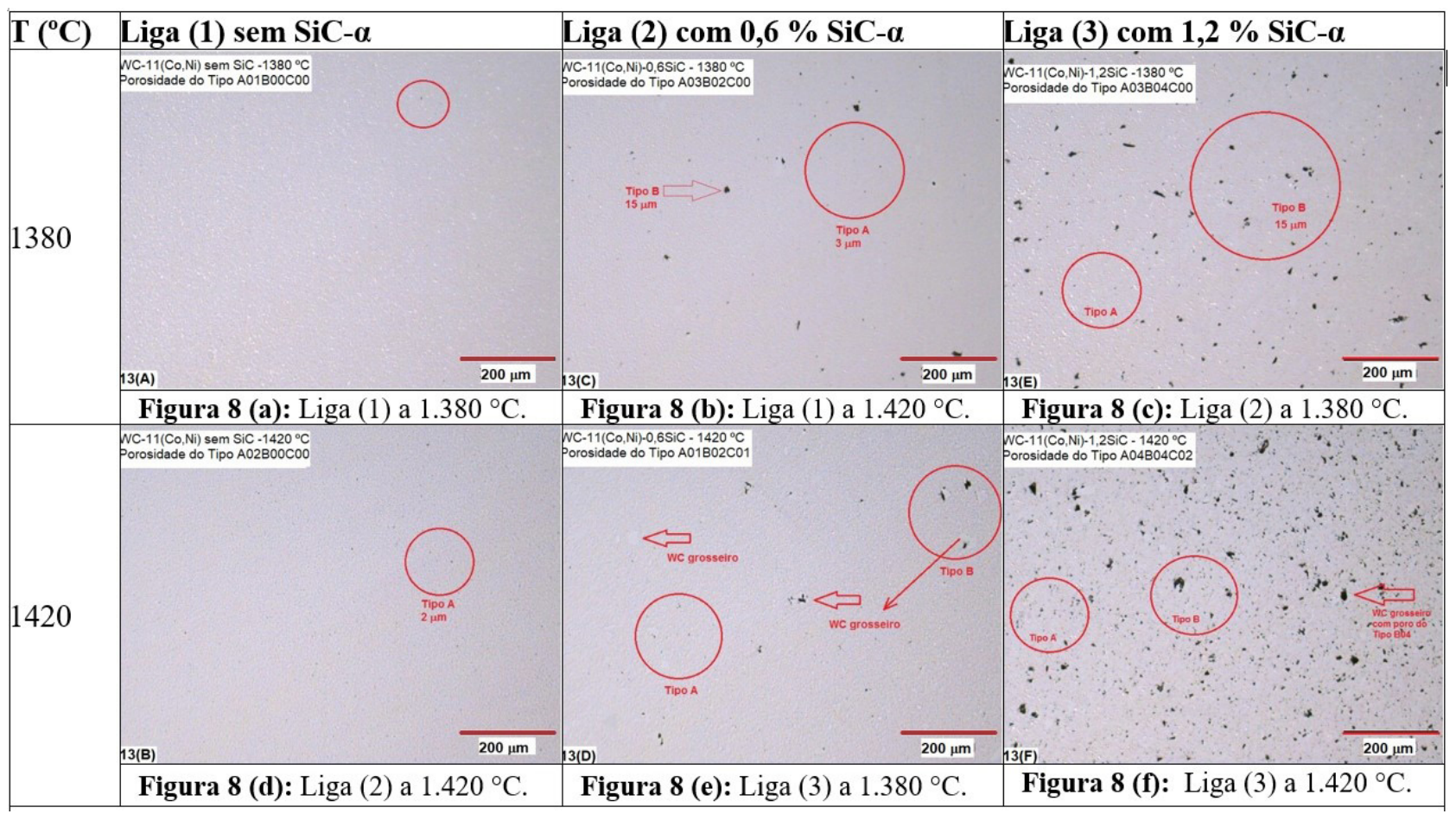

Figuras 8. (a) a (f): de porosidade aparente das ligas em função da adição de \% SiC- $\alpha$ e em função da temperatura de sinterização - sem ataque metalográfico - Microscópio ótico - ampliada 100x. 
carbono, e a partir de determinados teores pode fazer com que a liga de Co apresente uma estrutura cristalina cúbica estável em alta temperaturas, 760 a $980^{\circ} \mathrm{C}$ ou mesmo em temperatura ambiente. O ponto de fusão do $\mathrm{Ni}$ é de $1.455^{\circ} \mathrm{C}$,

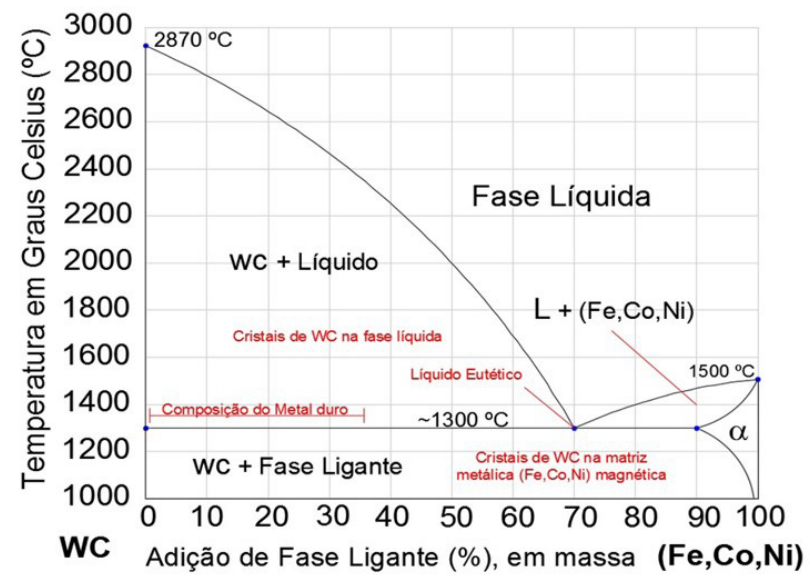

Figura 9. Sistema pseudo-binário (Fe, Co, Ni), adaptado [10].

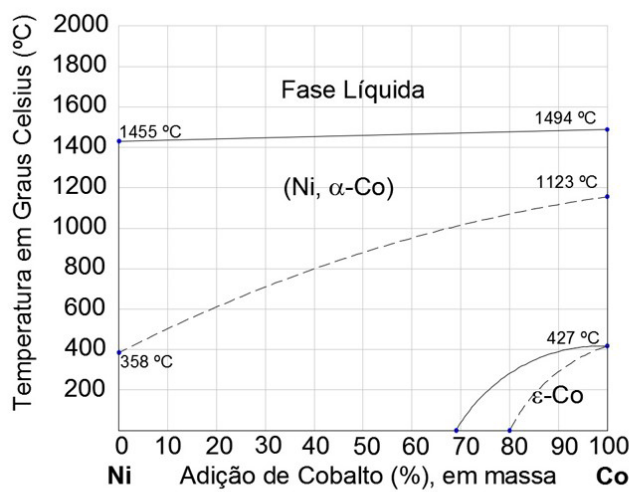

(a) sendo mais baixo do que o do Co, com $1.494^{\circ} \mathrm{C}$, no entanto, para se obter a densificação satisfatória é necessário aumentar o tempo e temperatura de sinterização [10-12].

A Figura 10b, apresenta o diagrama de fase de um binário Ni-Si, sujeito a formação do sólido policristalino Ni-Si. Para concentrações superiores a $10 \%$ em massa de silício no Ni tem-se uma solução sólida CFC ( $\mathrm{Si}, \mathrm{Ni}-\alpha)$. A solubilidade do Si no Ni é de $5 \%$ em massa para temperaturas abaixo de $700^{\circ} \mathrm{C}$ até a temperatura ambiente. A importância de destacar esse diagrama de fase $\mathrm{Ni}$-Si, é que pode haver tempo suficiente para permitir a difusão dos átomos de Si e $\mathrm{Ni}$, fusão congruente do $\mathrm{SiC}-\alpha$, podendo alterar a composição química na fase ligante e ocorrer a formação de fases, de $\mathrm{Si}$ em Ni- $\alpha$ e precipitando o carbono na forma livre [13].

Na Figura 11a é representada uma seção vertical do diagrama de fases W-Ni-C, correspondente a $10 \%$ em massa de Ni e na Figura 11b é representada uma seção vertical do diagrama de fases $\mathrm{W}-\mathrm{Co}-\mathrm{C}$, correspondente a $10 \%$ peso de Co e na Figura 11c é representada uma seção vertical do diagrama de fases $\mathrm{W}-\mathrm{Co}-\mathrm{Ni}$-C, todos calculados termodinamicamente $[11,14]$.

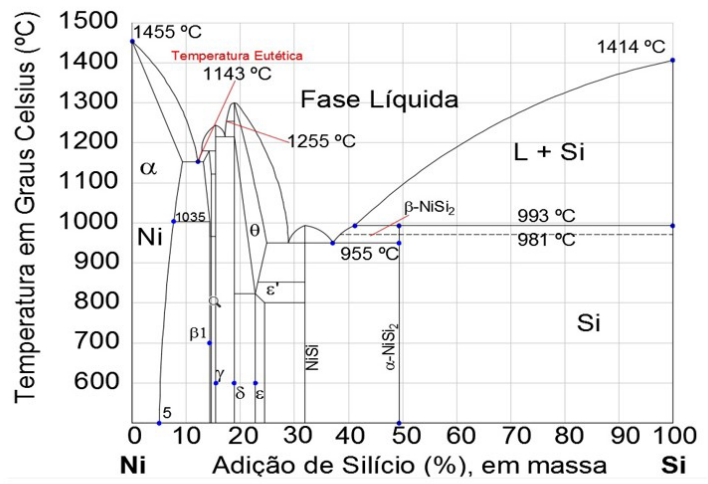

(b)

Figura 10. (a): Diagrama Ni-Co, adaptado [12,13]. (b): Diagrama Ni-Si, O composto intermediário que se funde congruentemente; adaptado [12,13].

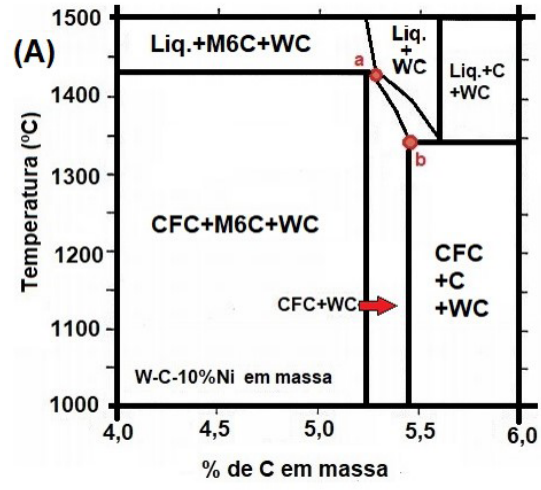

(a)

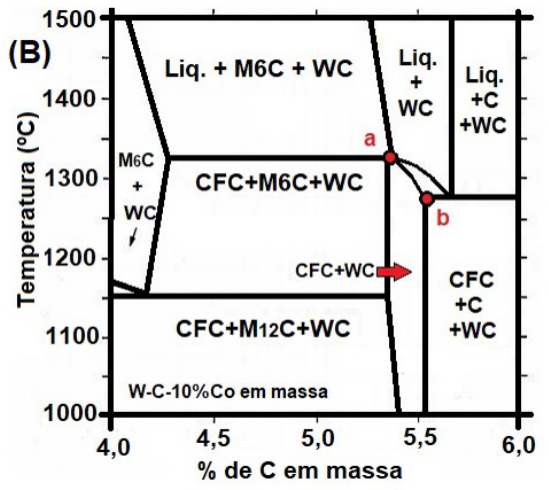

(b)

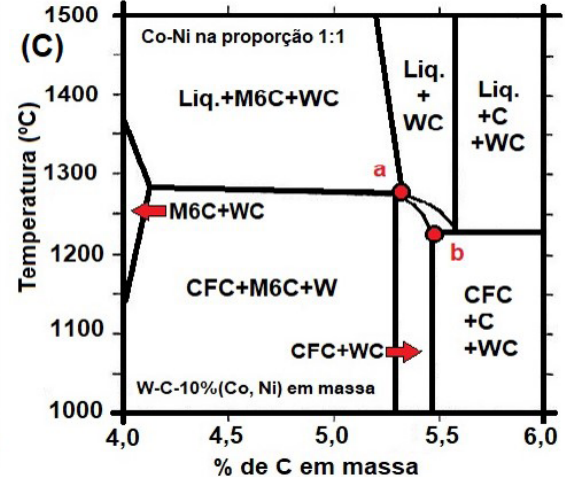

(c)

Figura 11.(a): Seção vertical do diagrama de fases W-C-10\%Ni em massa; (b) Seção vertical do diagrama de fases Co-W-C, para 10\% de Co e (c) seção vertical do diagrama de fases W-Co-Ni-C, para 10\% em massa (Co, Ni), 1:1 de Co-Ni. Adaptado [14]. 


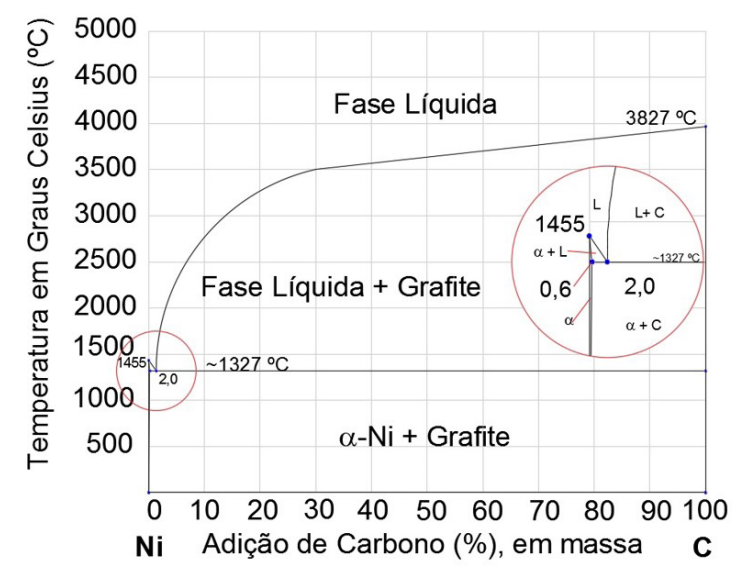

(a)

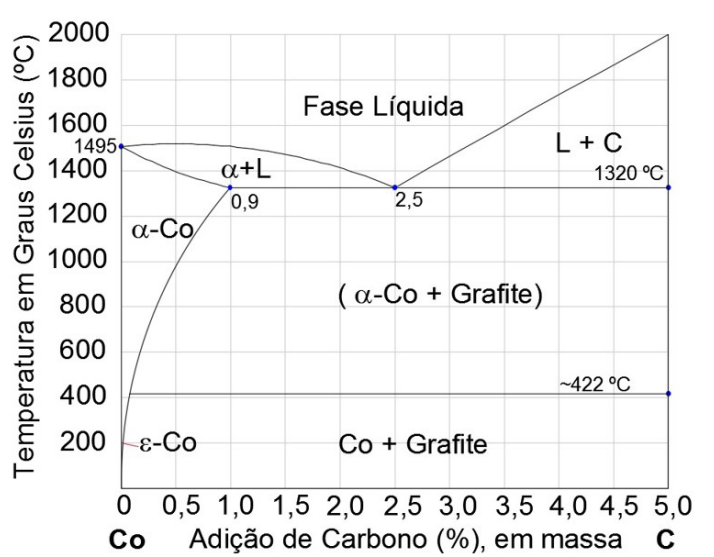

(b)

Figura 12. (a): Diagrama Ni-C, adaptado [13]. (b): Diagrama Co-C, adaptado [13].

Comparando os diagramas das Figuras $11 \mathrm{abc}$, para o sistema W-10(Co-Ni), são percebidas as consequências da substituição do Co pelo Ni. É possível visualizar que a largura da região bifásica $\mathrm{WC}+\mathrm{CFC}$ (estrutura cúbica de face centrada) permanece inalterada, mas a faixa de teores de carbono para sua formação desloca-se para valores menores. Verifica-se também que tal substituição implicou em um apreciável aumento das temperaturas de equilíbrio dos pontos eutético e peritético. Nos pontos "a" e "b", nas Figuras $11 \mathrm{abc}$, demonstram que com o aumento do carbono livre, ativa o crescimento do grão WC, facilitando em um crescimento anormal, bem como a morfologia e superfícies planas nas formas triangulares [14].

A substituição total do Co pelo $\mathrm{Ni}$ para as ligas WC, quando analisado o ponto "b" da Figura 11a em comparação com a Figura 11b, percebe-se um aumento das temperaturas de equilíbrio de 1.280 para $1.340{ }^{\circ} \mathrm{C}$. Além disso, é possível visualizar que a largura da região bifásica $\mathrm{WC}+\mathrm{CFC}$ (estrutura cúbica de face centrada) permanece inalterada, mas a faixa de teores de carbono para sua formação desloca-se para valores menores, essa substituição implica no aumento das temperaturas de equilíbrio dos pontos eutético e peritético $[11,14]$. Nas temperaturas de sinterização, a composição estequiométrica WC-10Ni, apresentando 5,52\% em massa de $\mathrm{C}$, encontra-se na região $\mathrm{WC}+$ líquido, porém, ao resfriar-se, há precipitação de grafita. Os pontos denotados por a e b, nas Figuras $11 \mathrm{abc}$ definem, respectivamente, os teores mínimo e máximo de carbono para ligas da região bifásica $\mathrm{C}+\mathrm{CFC}$, após a solidificação de equilíbrio. Para a proporção 1:1 de Co:Ni para WC-10(Co, Ni) o ponto de fusão da fase ligante, Figura 11(c), inicia-se aproximadamente $1.230^{\circ} \mathrm{C}$, no ponto "b" $[11,14]$.

O surgimento do carbono livre nas ligas (1), (2) e (3), também pode ser explicado nas Figuras $12 \mathrm{a}$ e $\mathrm{b}$.

No diagrama binário Ni-C, Figura $12 \mathrm{a}$, o ponto eutético permite a dissolução em solução líquida, do carbono livre para as ligas $\mathrm{W}-\mathrm{Ni}$, em até $2 \%$ em massa, formando um eutético com o $\mathrm{Ni}$ a uma temperatura de $1.327{ }^{\circ} \mathrm{C}$. Para o diagrama binário Co-C, Figura $12 \mathrm{~b}$, o ponto eutético, permite uma dissolução do carbono livre em até $2,5 \%$ para uma temperatura de $1.320{ }^{\circ} \mathrm{C}$. Durante a solidificação esses percentuais de dissolução do carbono diminuem, sendo $0,6 \%$ para o Ni, Figura 12 a e $0,9 \%$ para o Co Figura 12b. O crescimento anormal das partículas de WC, apresentada nas Figuras 3 a a $3 \mathrm{f}$ compromete o desempenho do produto, logo, deve ser evitado. Esse mecanismo não é completamente compreendido e muitas pesquisas sobre a prevenção do crescimento anormal dos grãos de WC estão sendo realizadas $[11,15,16]$.

Um aspecto crucial a ser considerado na produção dos carbonetos sinterizados, tanto para o WC como para o SiC- $\alpha$, é o controle do teor de carbono. A falta de carbono resulta em uma fase vítrea fragilizante denominada como fase eta $(\eta)$ e um excesso de carbono formará uma precipitação de grafite após o resfriamento, enquanto outros teores de $\mathrm{C}$ levarão à uma formação de fase, consistindo $\mathrm{M}_{6} \mathrm{C}$, mas são indesejáveis e prejudiciais para as ligas de metal duro, diminui propriedades mecânicas, como a tenacidade e o TRS. Portanto, o teor de carbono da liga sinterizada deve ser mantido dentro dos limites estreitos nos quais nem a fase eta e nem a grafite precipitam e isso só depende da composição geral do pó metálico fornecido. Em linhas de fabricação, o carbono livre deve ser controlado em intervalos de 0,01 a $0,05 \%$ em massa [17].

\section{Conclusões}

A mistura de Ni com o Co na proporção Ni-Co 1:1,5; o ponto de fusão inicia-se a $1.200{ }^{\circ} \mathrm{C}$ e há uma alteração na temperatura do eutético e peritético bem significativa. A substituição parcial do Co pelo Ni e do WC pelo $\mathrm{SiC}-\alpha$ em metais duros, são possíveis alternativas atraentes, devido à dependência da importação de matéria-prima e escassez no mercado mundial e isso é vital para desenvolver novas soluções para enfrentar o alto risco de restrições ao acesso desses elementos químicos. A substituição do Co é uma das tendências de pesquisas na área da sustentabilidade ambiental e o custo chega a $100 \%$ mais caro que o Ni. Com relação 
ao $\mathrm{WC}$, o custo da matéria-prima é dez vezes maior que o $\mathrm{SiC}-\alpha$. A presença de carbono livre na matéria-prima do pó de $\mathrm{SiC}-\alpha$ e a presença de porosidade nas ligas sinterizadas afetaram a resistência a ruptura transversal quando comparado com outras literaturas. Os particulados de WC cresceram de forma anormais e o carbono livre foi um dos contribuintes para esse fenômeno e deve ser evitado. Se faz necessário novos estudos com a adição de outros elementos químicos para contribuir na formação de carbonetos secundários ou inibir o crescimento de grãos anormais do WC e o controle de carbono livre com o máximo de $0,05 \%$ em massa. Verificar o comportamento da molhabilidade da fase ligante $(\mathrm{Co}$, $\mathrm{Ni}$ ), reavaliar o comportamento da dureza e a resistência da tensão de ruptura transversal.

\section{Agradecimentos}

Os autores agradecem à Brats, Imerys Minerals Fused-Treibacher Alodur ${ }^{\circledR}$ e a Universidade Federal Fluminense (UFF).

\section{Referências}

1 Roulon Z, Missiaen J-M, Lay S. Carbide grain growth in cemented carbides sintered with alternative binders. International Journal of Refractory Metals \& Hard Materials. 2019;86:105088.

2 Konyashin I, Klyachko L. History of cemented carbides in the Soviet Union. International Journal of Refractory Metals \& Hard Materials. 2015;49:9-26.

3 Toller L, Norgren S. Mechanisms of plastic deformation in WC-Co and WC-Ni-Fe turning inserts. In: Proceedings of the 19th Plansee Seminar. Austria: Plansee Group, 2017.

4 Correa EO, Santos JN, Klein AN. Microstructure, and mechanical properties of WC Ni-Si based cemented carbides developed by powder metallurgy. International Journal of Refractory Metals \& Hard Materials. 2010;28:572-575.

5 Lima TCF, Tenório JAS. Utilização de matéria-prima aglomerada na produção de carbureto de silício em escala piloto. REM. Revista Escola de Minas. 2011;64(3):341-345.

6 American Society for Testing and Materials. ASTM B294-17: standard test method for hardness testing of cemented carbides. West Conshohocken, PA: ASTM International; 2018.

7 International Organization for Standardization. ISO 3738-1. Hardmetals: rockwell hardness test (scale A) — Part 1: test method. Geneva: ISO; 1982.

8 American Society for Testing and Materials. ASTM E112: standard test methods for determining average grain size. West Conshohocken, PA: ASTM International; 2013.

9 American Society for Testing and Materials. ASTM B406-76: standard test method for transverse rupture strength of cemented carbides. West Conshohocken, PA: ASTM International; 2015.

10 Fernandes CM, Senos AMR. Cemented carbide phase diagrams: a review. International Journal of Refractory Metals \& Hard Materials. 2011;29:405-418.

11 Mannesson K. WC grain growth during sintering of cemented carbides: experiments and simulations [thesis]. Stockholm: Universitet Service US AB; 2011.

12 Okamoto H, Schlesinger ME, Mueller EM. ASM handbook Vol. 3: alloy phase diagrams. Ohio: ASM International; 2016.

13 Eisen WB, Ferguson BL, German RM, Lacocca R, Lee PW, Madan D, et al. ASM handbook Vol. 7: powder metal technologies, and applications metallurgy cermets and cemented carbides. Ohio: ASM International; 1998.

14 Guillermet AF. The Co-Fe-Ni-W-C phase diagram: a thermodynamic description and calculated sections for (Co-Fe-Ni) bonded cemented WC tools. Zeit. für Metall. 1989;2(80):83-94.

15 Roulon Z. Effect of binder on sintering and microstructure of cemented car-bides [thesis]. Grenoble: Université Grenoble Alpes; 2019.

16 Bjäreborn O. WC grain size distribution during sintering of WC-Co cemented carbides [thesis]. Sweden: Lund University; 2016.

17 García J, Ciprés VC, Blomqvist A, Kaplan B. Cemented carbide microstructures: a review. International Journal of Refractory Metals \& Hard Materials. 2018;80:40-68.

Recebido em: 31 Ago. 2020

Aceito em: 1 Abr. 2021 